\title{
Learning and Training Techniques in Fuzzy Control for Energy Efficiency in Buildings
}

\author{
J. Sedano ${ }^{\mathrm{a}}$, J.R. Villar ${ }^{\mathrm{b}}$, L. Curiel ${ }^{\mathrm{a}}$, E. Corchado ${ }^{\mathrm{c} *}$, and E. de la Cal ${ }^{\mathrm{b}}$ \\ ${ }^{a}$ Departament of Civil Engineering, University of Burgos, C/Villadiego, s/n, 09001, Burgos, Spain. E-mail: \\ jsedano@ubu.es \\ ${ }^{b}$ Department of Computer Science, University of Oviedo, Spain.E-mail:villarjose@univoi.es, delacal@uniovi.es \\ ${ }^{c}$ Department of Computer Science and Automation, University of Salamanca, Salamanca, Spain. E-mail: \\ escorchado@usal.es
}

\begin{abstract}
A novel procedure for learning Fuzzy Controllers (FC) is proposed that concerns with energy efficiency issues in distributing electrical energy to heaters in an electrical energy heating system. Energy rationalisation together with temperature control can significantly improve energy efficiency, by efficiently controlling electrical heating systems and electrical energy consumption. The novel procedure, which improves the training process, is designed to train the FC, as well as to run the control algorithm and to carry out energy distribution. Firstly, the dynamic thermal performance of different variables is mathematically modelled for each specific building type and climate zone. Secondly, an exploratory projection pursuit method is used to extract the relevant features. Finally, a supervised dynamic neural network model and identification techniques are applied to FC learning and training. The FC rule-set and parameter-set learning process is a multi-objective problem that minimises both the indoor temperature error and the energy deficit in the house. The reliability of the proposed procedure is validated for a city in a winter zone in Spain.
\end{abstract}

KEYWORDS: Computational Intelligence, Soft computing Systems, Mathematical and Identification Systems, Artificial Neural Networks, Non-linear Systems.

\section{Introduction}

The greater a society's prosperity, the greater the comfort that it might expect in its buildings and, consequently, the higher its consumption of electrical energy, which in great part explains government policies to promote the reduction of energy consumption. However, the reduction of energy consumption in the construction of buildings has not as yet been standardized [3, 9, 23]. For example, there are different approaches to heating systems in the literature that analyse the temperature control loop in order to improve heating system energy efficiency [26, 29, 33].

Soft Computing represents a broad range of techniques that can be used to solve inexact and complex problems [44]. It investigates, simulates, and analyzes very complex issues and phenomena in order to solve real-world problems [43]. In a previous study [43], Soft Computing was found valid for energy rationalization and temperature control by means of Fuzzy Controllers (FC). In this study, a novel procedure to to learn and train a FC for distributing electrical energy while controlling indoor temperatures is presented. As detailed in [43], the procedure firstly analyses the local building and heating regulations and market-related factors, in order to obtain suitable data for the learning and training step. The data set obtained in this way is processed, including a new feature selection and extraction stage based on Cooperative Maximum-Likelihood Hebbian Learning (CMLHL) [6] to extract the most relevant variables to model the indoor temperature.

Previous studies [24, 42] reflect the complexity of predicting the thermal dynamics of a building. In this study, a neural network approach based on system identification is shown to be valid for such modelling, which optimises the FC. Once the prediction model defines the thermal dynamics of the 
configuration, the learning and training of the FCs is carried out. Finally, the design stage ends using a Multi-Objective Simulated Annealing (MOSA) algorithm [40] to to learn and train the rule set and the parameter set, which minimises both the indoor temperature error and the electrical energy deficit in the house.

The structure of the paper is as follows. Section 2 introduces the different techniques used in the proposed procedure. Section 3 discusses the problem description. Section 4 details the multi-step procedure, while Section 5 presents the experiments and their results. Finally, the conclusions and comments on future lines of work are set out in the final section.

\section{Machine Intelligence techniques for optimising FC learning}

In Subsection 2.1 the feature selection and extraction method is introduced, while in Subsection 2.2 the specific technique for these tasks is detailed. Finally, in Subsection 2.3, the System Identification (SI) issues are discussed.

\subsection{Feature selection and extraction}

Feature Selection and extraction [4, 14, 27, 45] entail feature construction, space dimensionality reduction, sparse representations and feature selection, among others. They are all commonly used preprocessing tools in machine learning tasks, which include pattern recognition. Although researchers have grappled with such problems for many years, renewed interest has recently been shown in feature extraction.

The goal of using feature selection in this study is to analyse the internal structure of a data set representative of a case of study, in order to establish whether the data set is sufficiently informative the system has been excited in their operating ranges and it can be modeled successfully- and to choose a subset of input variables eliminating less important ones by using statistical methods, such as Principal Component Analysis (PCA) [20, 32] and unsupervised neural models, such as Cooperative Maximum Likelihood Hebbian Learning (CMHL) [8, 6].

\subsection{Data structure analysis using connectionist techniques}

The data analysis task is based on the use of a neural Exploratory Projection Pursuit (EPP) [11, 21] model called Cooperative Maximum Likelihood Hebbian Learning (CMLHL) [8, 6]. CMLHL, which is based on Maximum Likelihood Hebbian Learning (MLHL) [5, 7, 12], adds lateral connections [6, 8] which have been derived from the Rectified Gaussian Distribution [39]. The resultant net can find the independent factors of a data set but does so in a way that captures some type of global ordering in the data set.

Considering an N-dimensional input vector $(x)$, an M-dimensional output vector $(y)$ and with $W_{i j}$ being the weight (linking input $j$ to output $i$ ), CMLHL can be expressed $[6,8]$ as:

1. Feed-forward step:

$$
y_{i}=\sum_{j=1}^{\mathbf{N}} W_{i j} x_{j}, \forall i
$$

2. Lateral activation passing:

$$
y_{i}(t+1)=\left[y_{i}(t)+\tau(b-A y)\right]^{+}
$$

3. Feedback step:

4. Weight change:

$$
e_{j}=x_{j}-\sum_{i=1}^{M} W_{i j} y_{i}, \forall j
$$

$$
\Delta W_{i j}=\eta \cdot y_{i} \cdot \operatorname{sign}\left(e_{j}\right)\left|e_{j}\right|^{p-1} .
$$


Where: $\eta$ is the learning rate, $\tau$ is the "strength" of the lateral connections, $b$ the bias parameter, $p$ a parameter related to the energy function [5, 6], and $A$ a symmetric matrix used to modify the response to the data. The effect of this matrix is based on the relation between the distances among the output neurons.

\subsection{System Modelling Using Identification Algorithms}

The system Identification (SI) procedure includes several steps [28, 30]: selection of the models and their structure, their learning methods [17, 22, 41], identification and optimization criteria and the validation method. Validation ensures that the selected model meets the necessary conditions for estimation and prediction. Validation is typically carried out using three different methods: residual analysis $\varepsilon(t, \hat{\theta}(t))$, -by means of a correlation test between inputs, their residuals and their combinations; the final prediction error (FPE) estimated, as is explained, by Akaike [1] and finally a graphical comparison between desired outputs and the outcome of the models through simulation, with one (or k) steps ahead. The increase in computational capacity has allowed Artificial Neural Networks (ANNs) to become one of the most interesting soft computing paradigms used in SI. Some well-known model structures may be found in the literature $[2,13, \underline{19}]$.

When using ANN, the purpose of an identification process is to determine the weight matrix based on the observations $Z^{t}$, so as to obtain the relationships between the nodes in the network. The weight matrix is usually referred as $w, W$ or $\theta$. The supervised learning algorithm is then applied to find the estimator $\theta$, so as to obtain the identification criterion. Several well-known model structures are used when merging system identification with ANN. If the AutoRegressive with eXternal input model (ARX) is used as the regression vector, the model structure is called a Neural Network for ARX model (NNARX). Likewise, the Neural Network for Finite Impulse Response model (NNFIR), the Neural Network for Autoregressive Moving Average with eXternal input model (NNARMAX), and the Neural Network for Output Error model (NNOE), are also extensively used [30, 34, 36, 37].

\section{Optimising energy efficiency and temperature control}

In Spain, building and heating system regulations establish criteria for building design (issues such as building materials and insulation width, among others) and heating systems (nominal heating power for each installation). Moreover, they define up to five winter climate zones and five summer climate zones across the entire country. Building materials, insulation widths, and so on, are calculated accordingly to these < winter-zone, summer-zone> parameters.

In our opinion, more information than that contained in the regulations is needed when designing a heating system controller. Further market-related factors should also be included: the geometric design and orientation of a building, aesthetic aspects and its internal layout, all of which have a significant impact on thermal dynamics. Taken together, they define what is known as the topology of the building.

In a previous study, a two-stage method for calculating a FC that would control an electrical energy heating system was proposed [43]. In the first stage (called the design stage), FC learning took place for each topology; in the second stage (called the run stage), the FC was exploited for the corresponding climate zone. The design stage included several steps. Firstly, realistic building information and historical meteorological data for each climate zone were gathered and used in a simulation that ran on HTB2 software [25]. Heating and ventilation systems were also designed and other profiles were estimated in relation to occupancy, lighting, small power devices, ventilation, and set-point temperatures, among other aspects.

The HTB2 software produced a data set containing the evolution of indoor temperature and the heating power requirements for each room, among others. This data set was manually post-processed in order to be used in the learning of FCs. Then, the post-processed data set was used for training a polynomial model to estimate the room temperature evolution given the instantaneous heating power. In this case the polynomial model was fitted using Simulated Annealing. 
Finally, the temperature model and the post-processed data set were used for learning the FC for a specific climate zone using Multi-objective Simulated Annealing (MOSA) [40]. The two objectives to optimise were the temperature error and the electrical energy deficit. The latter measure was calculated as the difference between the required heating power estimated with the HTB2 and the heating power proposed by the FC.

This previous study a better electric energy distribution scheme was obtained, but its main strong point was that the FC would reduce the heating energy, thereby improving energy efficiency in the installation. However, two important drawbacks were identified: the proposed room temperature estimation did not behave with the required precision and the post-processing stage had to be analysed to arrive at a better procedure. Solutions to these two drawbacks were approached in this research Firstly, a better model for the room temperature is proposed. Secondly, the post-processing stage has been automated. Both enhancements are to be detailed in the next Section.

\section{A multi-step procedure for detecting thermal dynamics in buildings}

The results from subsequent studies [34, 37, 38] that used a procedure for detecting thermal insulation failures in buildings in operation provide a clue for solving the two above-mentioned drawbacks: the use of CMLHL to find the most relevant variables for estimating in-room temperature evolution.

Using these ideas, the four-step procedure detailed in previous section has been improved. Two of the four steps remain unchanged in the new approach: the initial step in which the meteorological data and the realistic data and profiles are used in the HTB2 simulation tool, and the final step for learning and training the FC using the MOSA algorithm. Nevertheless, a new post-processing step is introduced using CMLHL as a dimensionality reduction technique to choose the most relevant features in order to determine the indoor temperature. Finally, the Simulated Annealing step for training a polynomial model was replaced with an ANN model, as proposed in [34, 37, 38]. The differences between the two methods are depicted in Figure 1.

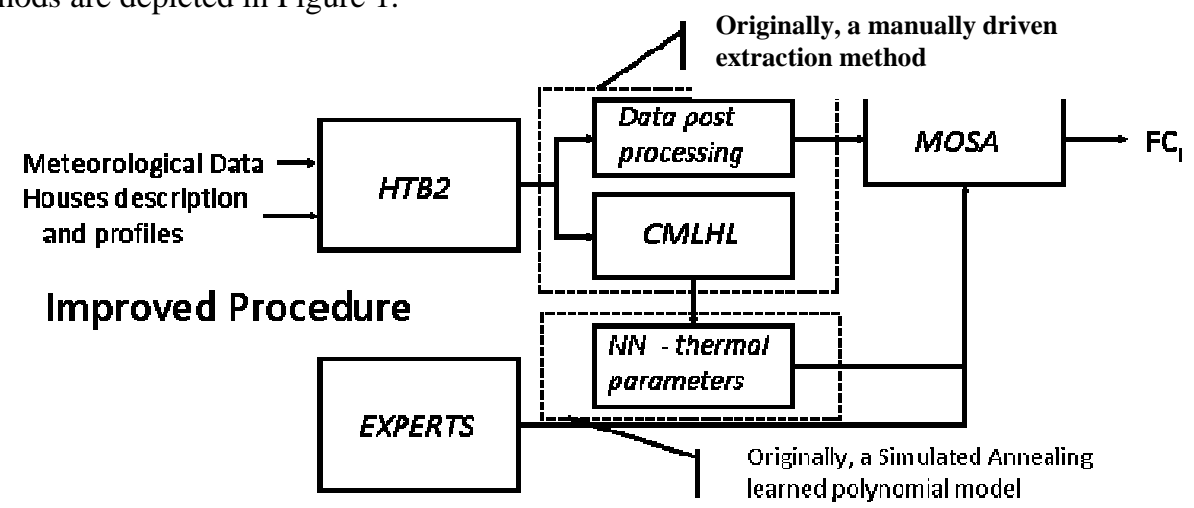

Figure 1. The schema of the procedure learning FCs. The HTB2 outcome was manually post-processed -in a previous study- to obtain two data sets, one for the training of a room temperature evolution and the second for the learning of the FC using the MOSA algorithm. In the new approach, CMLHL is applied to determine and extract the most relevant features for determining the room temperature evolution, which is now modelled with a Neural Network.

The following sub-sections deal with the description of each step. In the next subsection, the generation of the thermal dynamics data set is described. Sub-Section 4.2 presents the CMLHL step, while in Sub-Section 4.3 the procedure to obtain the indoor temperature prediction model is detailed. Finally, the learning of the FCs is outlined. 


\subsection{Thermal dynamics data gathering by means of simulation.}

Certain variables and data sets should be gathered to simulate the thermal behaviour of a building. This data includes the building topology -the inner distribution and orientation of the building under analysis-, the climate zone defined in specific regulations, the building materials that comply with local regulations according to the chosen climate zone, the meteorological data for the climate zone and the simulated time period -solar radiation, outdoor temperature, wind speed and so on, which are generally available from weather stations in the climate zone-, and realistic profiles for heating, lighting, small power devices, occupancy and ventilation. In this study, the procedure is applied in Spain, where the regulations establish five winter/summer zones, from E1 (more severe climate zones) to A3 (gentler climate zone).

Having defined and/or gathered these data sets, the chosen simulation tool is then applied to obtain the output data. The output from HTB2 is a data set that includes data on the evolution of the indoor temperature and heating power for each space in the building according to its expected operating conditions. The typical values that each variable could take for an E winter climate zone of maximum severity in Spain -i.e. the cities of León, Burgos or Soria among others- are shown in Table 1.

Table 1. Typical values of each variable in an E winter climate zone city in Spain

\begin{tabular}{|l|c||l|}
\hline \multicolumn{1}{|c|}{ Variable (Units) } & $\begin{array}{c}\text { Range of } \\
\text { values }\end{array}$ & \multicolumn{1}{|c|}{ Transmittance level $\left(\mathbf{W} / \mathbf{m}^{2} \mathbf{K}\right)$} \\
\cline { 1 - 2 } Air temperature of the house $\left({ }^{\circ} \mathrm{C}\right), \mathrm{y}_{1}(\mathrm{t})$. & 14 to 24 & -External cavity wall: 0.54 \\
\hline Exterior air temperature in February $\left({ }^{\circ} \mathrm{C}\right), \mathrm{u}_{1}(\mathrm{t})$. & 1 to 7 & $\begin{array}{l}\text {-Double glazing: } 2.90 \\
\text {-Floor/ceiling: } 1.96\end{array}$ \\
\hline Heater gain $(\mathrm{w}), \mathrm{u}_{2}(\mathrm{t})$. & 0 to 4,250 & $\begin{array}{l}\text {-Party wall between buildings: } 0.96 \\
\text {-Others party wall: } 1.050\end{array}$ \\
\hline Small power and occupancy gain $(\mathrm{w}), \mathrm{u}_{3}(\mathrm{t})$. & 0 to 1,200 & -Internal partition: 2.57 \\
\hline Lighting gain $(\mathrm{w}), \mathrm{u}_{4}(\mathrm{t})$. & 0 to 500 & \\
\hline Ventilation gain $\left(\mathrm{m}^{3} / \mathrm{min}\right), \mathrm{u}_{5}(\mathrm{t})$. & 0.5 to 7.5 & \\
\hline
\end{tabular}

\subsection{Selection of the relevant features}

PCA and CMLHL (Section 2) are both applied to this real-life problem and are instrumental in identifying the internal structure of the data. In this procedure, the data set gathered in the previous step is analysed. The objective is to find the relationships between the input variables with respect to the indoor temperature. CMLHL was used to detect the dependent relations and to choose the most relevant features. The output of this step is a new data set of a reduced dimension that only contains the data that corresponds to those features for which a relationship with the indoor temperature has been found.

\subsection{Modelling normal building operation}

Having extracted the relevant variables and their transformations from the thermal dynamics data, then a model to fit the normal building operation has to be obtained in order to identify bias in the room temperature, which is in the end used for failure detection. The different model learning methods used in this study were implemented in Matlab@ [31].

The model structures mentioned in Section 2.3 were analyzed in order to obtain the models that best suited the dataset. The Akaike Information Criterion (AIC) was used to obtain the best degree of the model and its delay for each model structure. Up to thirty-four different combinations of nonlinear model structures and optimization techniques were considered -including the Levenberg-Marquardt method, the batch version of the back-propagation algorithm and the recursive Gauss-Newton method for the NNARX, NNFIR, NNARMAX and NNOE models $[10,18,28]-$.

Three different residual analyses based on cross correlation were carried out: residual analysis between the residual $\hat{R}_{\varepsilon}^{N}(\tau)$, between the residual and the input $\hat{R}_{\varepsilon u}^{N}(\tau)$ and the non-linear residual correlation $\hat{R}_{\varepsilon^{2} u^{2}}^{N}(\tau)$ [28].

Moreover, several different indexes were used to validate the models. The indexes are well-known and widely-used measures in system identification [28, 30, 35]: the percentage representation of the estimated model; the graphical representation for the one-step prediction $-\hat{y}_{1}(t \mid m)-$; for ten-step 
prediction $-\hat{y}_{10}(t \mid m)$ - and for the $\infty$-step prediction $-\hat{y}_{\infty}(t \mid m)$-, versus the measured output $-y_{1}(t)$-; the loss function or error function $(\mathrm{V})$; the generalization error value and the Final Prediction Error (FPE), calculated as the average generalization error value computed with the estimation dataset.

The percentage representation of the estimated model is calculated as the normalized mean error for the one-step prediction (FIT1), for the ten-step prediction (FIT10) and with the $\infty$-step prediction (FIT). The FIT is widely used as a simulation technique in classical system identification. The loss function or error function (V) is the numeric value of the mean squared error (MSE) that is computed with the estimation data set. Finally, the generalization error value is the numeric value of the normalized sum of squared errors (NSSE) that is computed with the validation data set [28, 36].

\subsection{Learning the FC using multi-objective techniques}

The last step in the procedure is to learn the FC. For such tasks, data from the HTB2 output data set and the Neural Network model obtained from the previous step are used with recurrent simulations. The MOSA algorithm is used to evolve the FC from that proposed by the experts; both rules and parameters are developed. The MOSA is used due to the fact that evaluating an FC is a multi objective problem as two objectives are optimized: the minimization of the recurrent error and the minimization of the electrical energy deficit. The former aims to produce high quality models while the latter is employed to obtain a better electrical energy distribution among the electrical heaters [43]. The model which is closer to the origin is chosen.

\section{Experimentation and results}

As the results for the other parts of the procedure have yet to be documented [43], only the results that consider the new steps are presented: the CMLHL analysis for feature selection and extraction and the generation of the steps in the Neural model.

Some realistic situations have been considered in order to validate the procedure. A building in the E winter zone, in the city of León is used as the actual building. As shown in Figure 1, this building was parameterised and the HTB2 simulation tool was used to gather the data set. This initial data set was analysed, in order to select the features that best describe the relationships with the indoor temperature.

As may be seen in Figure 2, PCA (Figure 2.a) and CMLHL (Figure 2.b) have found a clear internal structure in the dataset. Both methods identified heater output and small power and occupancy as relevant variables. The CMLHL projections give us more information because it has recognised the lighting as another important variable.

Having analysed the results obtained with the CMLHL model (Figure 2.b), the conclusion was that CMLHL identified two different clusters ordered by lighting. Inside each cluster there are further classifications by small power and occupancy and heater output, and the dataset may be said to have an interesting internal structure. When the dataset is considered sufficiently informative, the third step in the process begins. This step performs an accurate and efficient optimization of the heating system model to detect thermal insulation failures in the building, through the application of several conventional modelling systems. 


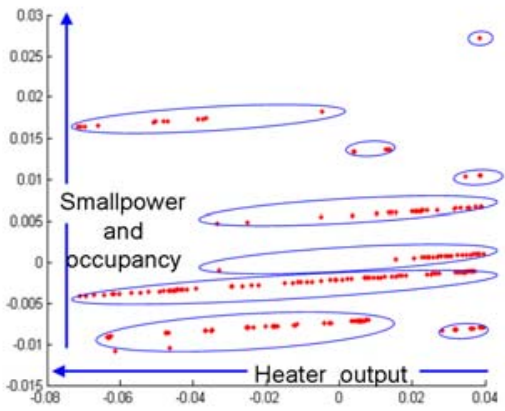

Fig. 2.a. PCA projections.

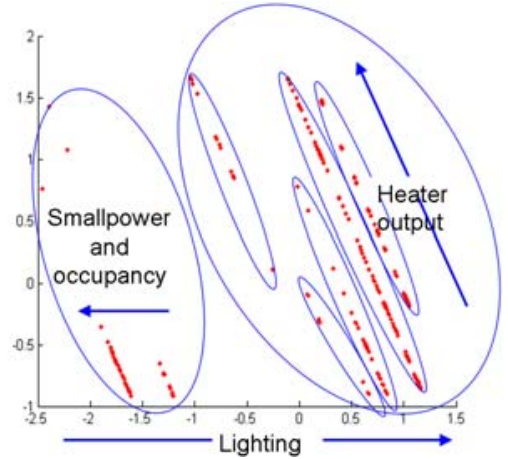

Fig. 2.b. CMLHL projections.

Figure 2. PCA projections (Figure 2.a) and CMLHL projection (Figure 2.b) after 2500 iterations using a learning rate of 0.05 , 3 output neurons, $\mathrm{p}=0.1$ and $\tau=0.2$.

Thus, a dynamic ANN was used to monitor the thermal dynamics of the building. The objective was to find the best suite of polynomial model orders [ $n_{a} n_{b 1} n_{b 2} n_{b 3} n_{b 4} n_{b 5} n_{c} n_{d} n_{f} n_{k 1} n_{k 2} n_{k 3} n_{k 4} n_{k 5}$, which is the optimal architecture of ANN. Using the data set from the previous stage and the Optimal Brain Surgeon (OBS) $[15,16]$ network pruning strategy to remove superfluous weights, the best suite model was found from the residual analysis. Table 2 shows the estimation and prediction characteristics and qualities of the chosen ANN, along with their indexes.

The graphic representations of $\hat{y}_{1}(t \mid m)$ for indoor temperature of the house $-y_{1}(t)$-are shown in Figure 3.a for a pruned NNARX model structure. The $\mathrm{x}$-axis shows the number of samples used in the estimation and validation of the model and the $y$-axis represents the normalized output variable range, which is the normalized indoor temperature of the house. The estimation and validation data sets include 2000 and 1126 samples, respectively, and have a sampling rate of 1 sample/minute. Figure 3.b indicates the final neural network structure chosen for indoor temperature prediction, both of which are polynomial model orders. These orders specify the inputs to the ANN -five variables for a full connected- and the indices of the orders represent each of the thermal system inputs.

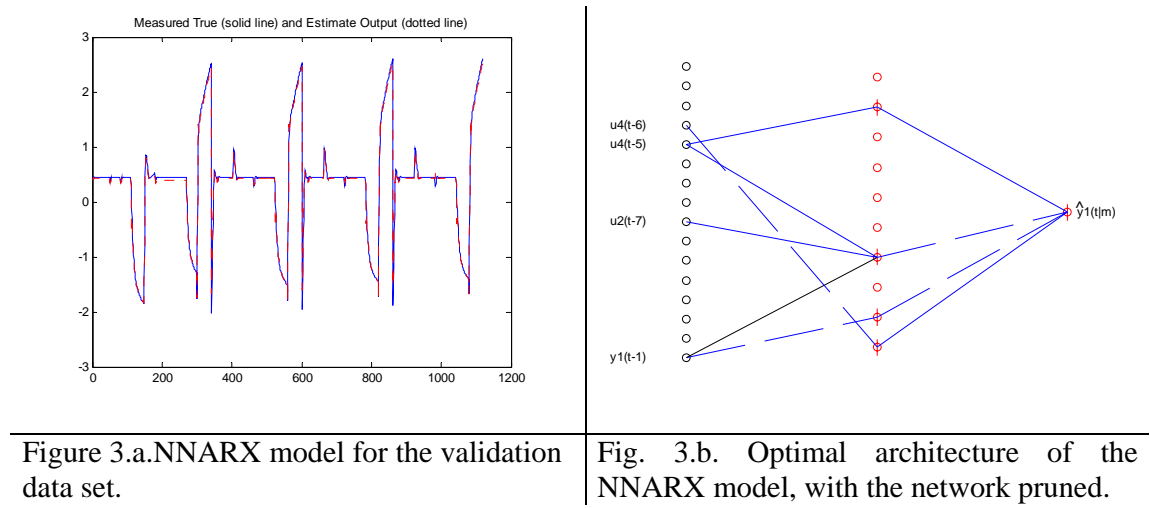

Figure 3. In Figure 3.a the real measure is compared with the validation data. The actual output (solid line) is graphically presented with one-step-ahead prediction (dotted line). In Figure 3.b the optimal architecture of the NNARX model, with the network pruned, is presented. Positive weights are indicated by solid lines, while a dashed line represents a negative weight. A vertical line through the neuron represents a bias.

From Figure 3, it can be concluded that the pruned network NNARX model is able to predict the behaviour of the indoor temperature of the house and is capable of modelling more than $93 \%$ of the actual measurements. In Table 2, the value of the indexes obtained for the NNARX model are shown. 
These models not only present a lower loss function (V) and error values (NSSE and FPE), but also a higher system representation index value (FIT1).

Once the thermal dynamic -in the building- of the configuration is defined, the learning and training of the FCs is carried out. Firstly, the basic FC established by the experts is optimized by a rule learning process. Secondly, the training of the fuzzy sets of the rule set is carried out. The objective to be accomplished is to minimise both the temperature error and the energy deficit in the building. This is a multi-objective problem which used the multi-objective simulated annealing algorithm presented in [40].

Table 2. The value of the quality indexes obtained for the proposed model. FIT1, V, NSSE and FPE stand for the graphical representation percentage, the loss function error, the normalised sum of squared error and the final prediction error.

\begin{tabular}{|c|c|}
\hline Model & Indexes \\
\hline 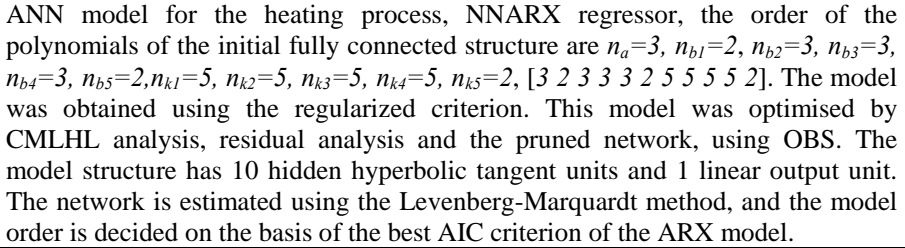 & $\begin{array}{l}\text { FIT1: } 93.42 \% \\
\text { V: } 0.0056 \\
\text { FPE: } 0.102 \\
\text { NSSE: } 0.0036\end{array}$ \\
\hline
\end{tabular}

\section{Conclusions and future work}

Energy distribution and temperature control is an essential component of energy efficient heating systems in buildings. In this research, an improved design stage for learning and training a FC has been presented.

Exploratory Pursuit methods have been used in feature selection and extraction, to search for the most informative feature set and data structure. CMLHL was able to identify the internal structure of the data and to choose the feature set. Finally, different techniques were applied to obtain a suitable prediction model, which was used for the prediction of indoor temperature.

The results show a new promising method for learning FCs. The use of CMLHL with dynamic neural modelling -NNARX- improves on the response obtained from the methods used in earlier studies. Future work will include experimenting with the entire procedure and testing the FC in real world installations, in order to validate the overall proposal.

\section{Acknowledgments}

This work has been partially supported through the Junta of Castilla and León (JCyL): [BU006A08], the Spanish Ministry of Science and Innovation [PID 560300-2009-11], the Spanish Ministry of Education and Innovation [CIT-020000-2008-2], the Spanish Ministry of Science and Technology [TIN2008-06681-C06-04] and Grupo Antolin Ingenieria, S.A., within the framework of MAGNO2008 - 1028.- CENIT also funded by the same Government Ministry. We would like to extend our thanks to Phd. Magnus Nørgaard for his freeware version of Matlab Neural Network Based System Identification Toolbox

\section{References}

[1] H. Akaike. Fitting Autoregressive Models for Prediction. Annals of the Institute of Statistical Mathematics, 20:425-439, 1969.

[2] S.D. Bekiros and D.A. Georgoutsos. Evaluating direction of change Forecasting: Neurofuzzy Models versus Neural Networks. Mathematical and Computer Modelling, 46: 38-46, 2007.

[3] M. Bojic, M. Despotovic, J. Malesevic, and D. Sokovic. Evaluation of the impact of internal partitions on energy conservation for residential buildings in Serbia. Building and Environment, 42(4):1644-1653, 2007.

[4] C. Chen and J. Yang. Hidden Space-Based Nonlinear Discriminant Feature Extraction Method. International Journal of Computer Mathematics, 84(9):1299-1308, 2007. 
[5] E. Corchado, D.MacDonald, and C. Fyfe. Maximum and Minimum Likelihood Hebbian Learning for Exploratory Projection Pursuit. Data Mining and Knowledge Discovery, 8(3):203-225, 2004.

[6] E. Corchado and C. Fyfe. Connectionist Techniques for the Identification and Suppression of Interfering Underlying Factors. International Journal of Pattern Recognition and Artificial Intelligence, 17(8):1447-1466, 2003.

[7] E. Corchado and C. Fyfe. Orientation Selection Using Maximum Likelihood Hebbian Learning. International Journal of Knowledge-Based Intelligent Engineering Systems, 7(2):11-30, 2003.

[8] E. Corchado, Y. Han, and C. Fyfe. Structuring global responses of local filters using lateral connections. Journal of Experimental \& Theoretical Artificial Intelligence, 15(4):473-487, 2003.

[9] B. Farhanieh and S. Sattari. Simulation of energy saving in Iranian buildings using integrative modelling for insulation. Renewable Energy, 31(4):417-425, 2006.

[10] R. Fletcher. Practical Methods of Optimization. Wiley \& Sons, Chichester, UK, $2^{\text {nd }}$ edition, 1987.

[11] J.H. Friedman and J.W. Tukey. Projection Pursuit Algorithm for Exploratory Data-Analysis. IEEE Transactions on Computers, 23(9):881-890, 1974.

[12] C. Fyfe and E. Corchado. Maximum Likelihood Hebbian Rules. In Proceedings of the $10^{\text {th }}$ European Symposium on Artificial Neural Networks (ESANN 2002), pp. 143-148, Bruges, Belgium, 2002. D-side Publishers.

[13] M.M. Gupta and D.H. Rao. On the Principles of Fuzzy Neural Networks. Fuzzy Sets and Systems, 61(10):1 - 18, 1994.

[14] I. Guyon and A. Elisseeff. An Introduction to Variable and Feature Selection. Journal of Machine Learning Research, Special Issue on Variable and Feature Selection, 3:1157-1182, 2003.

[15] L.K. Hansen and M.W. Pedersen. Controlled Growth of Cascade Correlation Nets. In International Conference on Artificial Neural Networks (ICANN1994), pp. 797-800, Italy, 1994. Eds. M. Marinaro and P.G. Morasso.

[16] B. Hassibi and D.G. Stork. Second Order Derivatives for Network Pruning: Optimal Brain Surgeon. In Advances in neural Information Processing System 5, pp. 164-171, San Mateo, CA, USA, 1993. Eds. S.J. Hanson et al.

[17] X. He and H. Asada. A new method for identifying orders of input-output models for nonlinear dynamic systems. In Proceedings of the American Control Conf., S.F., pp. 2520-2523, California, 1993.

[18] J. Hertz, A. Krogh, and R.G. Palmer. Introduction to the Theory of Neural Computation, Vol. 1. Addison-Wesley, Santa Fe Institute Studies In The Sciences Of Complexity, 1991.

[19] K. Hornik, M. Stinchcombe, and H. White. Multilayer Feedforward Networks are Universal Approximators. Neural Networks, 2(5):359-366, 1989.

[20] H. Hotelling. Analysis of a Complex of Statistical Variables Into Principal Components. Journal of Education Psychology, 24:417-444, 1933.

[21] A. Hyvarinen. Complexity Pursuit: Separating Interesting Components from Time Series. Neural Computation, 13(4):883898, 2001.

[22] N. Kofidis, A. Margaris, K. Diamantaras, and M. Roumeliotis. Blind System Identification: Instantaneous Mixtures of $\mathrm{n}$ Sources. International Journal of Computer Mathematics, 85(9):1333-1340, 2008.

[23] C. Koroneos and G. Kottas. Energy consumption modeling analysis and environmental impact assessment of model house in Thessaloniki-Greece. Building and Environment, 42(1):122-138, 2007.

[24] F. Lai, F. Magoulés, and F. Lherminier. Vapnik's learning theory applied to energy consumption forecasts in residential buildings. International Journal of Computer Mathematics, 85(10):1563-1588, 2008.

[25] P.T. Lewis and P.K. Alexander. A Flexible Model for Dynamic Building Simulation: HTB2. Building and Environment, 1:7-16, 1990.

[26] C.Y. Lin and C.Y. Juan. Double-loop temperature control of an on-off heating system. In Proceedings of the 3rd WSEAS international conference on Circuits, systems, signal and telecommunications. CISST'09, pp. 154-158, Stevens Point, Wisconsin, USA, 2009. World Scientific and Engineering Academy and Society (WSEAS).

[27] H. Liu and L. Yu. Toward Integrating Feature Selection Algorithms for Classification and Clustering. IEEE Educational Activities Department, 17(4):491-502, 2005.

[28] L. Ljung. System Identification. Theory for the User. Prentice-Hall, Upper Saddle River, N.J., USA, $2^{\text {nd }}$ edition, 1999.

[29] N. Nassif and S.Moujaes. A cost-effective operating strategy to reduce energy consumption in a HVAC system. International Journal of Energy Research, 32(6):543-558, 2008.

[30] M. Nørgaard, O. Ravn, N.K. Poulsen, and L.K. Hansen. Neural Networks for Modelling and Control of Dynamic Systems. Springer-Verlag, London U.K., 2000.

[31] M. Nørgaard. Neural network Based System Identification Toolbox. Technical Report 00-E-891, Department of Automation, Technical University of Denmark, 2000.

[32] K. Pearson. On Lines and Planes of Closest Fit to Systems of Points in Space. Philosophical Magazine, 2(6):559-572, 1901.

[33] D. Popescu, C. Ciufudean, and A. Ghiaus. Specific aspects of design of the automated system for heating control that accounts for heat losses through the building's envelope. In Proceedings of the 13th WSEAS International Conference on Systems. ICS'09, pp. 352-356, Stevens Point, Wisconsin, USA, 2009. World Scientific and Engineering Academy and Society (WSEAS).

[34] J. Sedano, E. Cal, L. Curiel, J.R. Villar, and E. Corchado. Soft Computing for detecting Thermal Insulation Failures in Buildings. In Proceedings of 9th International Conference on Computational and Mathematical Methods in Science and Engineering, Vol. 4, pp. 1392-1402, Gijón, Spain, June 2009.

[35] J. Sedano, E. Corchado, L.Curiel, J.R. Villar, and P.M. Bravo. The Application of a two-step AI Model to an Automated Pneumatic Drilling Process. International Journal of Computer Mathematics, 86(10-11):1769-1777, 2009.

[36] J. Sedano, L. Curiel, E. Corchado, E. de la Cal, and J.R. Villar. A Soft Computing Based Method for Detecting Lifetime Building Thermal Insulation Failures. Integrated Computer-Aided Engineering, IOS Press, 17(2):103-115, 2010. 
[37] J. Sedano, J.R. Villar, L. Curiel, E. de la Cal, and E. Corchado. Improving Energy Efficiency in Buildings using Machine Intelligence. In E. Corchado and H. Yin, editors, Intelligent Data Engineering and Automated Learning. IDEAL 2009, Vol. 5788, pp. 773-782. Springer Berlin / Heidelberg. Lecture Notes in Computer Science, 2009.

[38] J. Sedano, J.R. Villar, L. Curiel, E. de la Cal, and E. Corchado. Modelling of heat Flux in Building using Soft-computing Techniques. In E. Corchado and H. Yin, editors, $23^{\text {th }}$ International Conference on Industrial, Engineering \& Others Applications of Applied Intelligent Systems, IEA-AIE, Vol. 6098, pp. 636-645. Springer. Lecture Notes in Artificial Intelligent, 2010.

[39] H.S. Seung, N.D. Socci, and D. Lee. The Rectified Gaussian Distribution. Advances in Neural Information Processing Systems, 10:350-356, 1998.

[40] L. Sánchez and J.R. Villar. Obtaining transparent models of chaotic systems with multi-objective simulated annealing algorithms. Information Sciences, 178(4):952-970, 2008.

[41] P. Stoica and T. Söderström. A Useful Parametrization for Optimal Experimental Design. In IEEE Transactions on Automatic Control, $A C-27,1982$.

[42] J.R. Villar, E. de la Cal, and J. Sedano. Minimizing Energy Consumption in Heating Systems under Uncertainty. In Lecture Notes in Artificial Intelligence: Hybrid Artificial Intelligence Systems, Vol. 5271, pp. 583-590. Springer, 2008.

[43] J.R. Villar, E. de la Cal, and J. Sedano. A fuzzy logic based efficient energy saving approach for domestic heating systems. Integrated Computer-Aided Engineering, 16(2):151-163, 2009.

[44] L.A. Zadeh. Soft computing and fuzzy logic. IEEE Software, 11(6):48-56, 1994.

[45] J. Zhang, T. Jiang, B. Liu, X. Jiang, and H. Zhao. Systematic Benchmarking of Microarray Data Feature Extraction and Classification. International Journal of Computer Mathematics, 85(5):803-811, 2008. 